\title{
Corrective Four-fold Osteotomy of The Forearm Performed with 3D-Planning and Patient-Specific Guides
}

\author{
Andrea Frey and Andreas Schweizer* \\ Department of Orthopaedic Surgery, University of Zurich, Uniklinik Balgrist, Switzerland
}

*Corresponding author: Andreas Schweizer, Department of Orthopaedic Surgery, University of Zurich, Uniklinik Balgrist, Switzerland

\section{ARTICLE INFO}

Received: 粜 November 25, 2019

Published: 慧December 03, 2019

Citation: Andrea Frey, Andreas Schweizer. Corrective Four-fold Osteotomy of The Forearm Performed with 3D-Planning and Patient-Specific Guides. Biomed J Sci \& Tech Res 23(3)-2019. BJSTR. MS.ID.003913.

\section{ABSTRACT}

Preoperative three-dimensional planning methods for the use of patient specific implants have been extensively described. In this report, we describe a corrective fourfold osteotomy nof the radius and ulna in the management of a severely deformed forearm with a limited ROM of Pro-Sup of $0-20-70^{\circ}$ using patient-specific guides for accurate spatial positioning and fixation following pre-operative three-dimensional planning. Twenty months postoperatively the patient shows almost normal function and range of motion of.

Keywords: Corrective Osteotomy; Patient-Specific Guide

\section{Introduction}

Malunion following a distal forearm fracture, especially the radius, is a common complication that may treated by corrective osteotomy surgery [1-3]. Accurate reconstruction is essential as a statistically significant relationship has been found between mal-positioning and clinical outcome [4,5]. Additionally, it has been demonstrated that standard anatomical plates may lead to considerable positioning errors in individual patients [6]. Threedimensional (3D) planned patient-specific guides and implants are increasingly valuable, allowing for precise corrective osteotomies of complex multiplanar forearm deformities $[7,8]$. In this report, we describe the management of a severely malunited forearm fracture previously inadequately treated via corrective osteotomy. In this case, a novel patient-specific plate, which fits the bone geometry and accurately was used to restore bone alignment.

\section{Case Report}

The 25-year-old female patient presented in our outpatient clinic with left-sided dominant forearm malunion following radius and ulna shaft fracture at 14 years of age. The fracture was treated without initial reposition in a splint cast. Therefore, the patient developed a pronounced reduction in pro- and supination despite intensive occupational therapy. Five years post-traumatically, corrective osteotomy of the forearm was performed in an external clinic in order to improve range of motion. In the surgical report intraoperative adjustment of the preoperative planning was necessary to ameliorate supination of the forearm. Following surgery increasingly limited pronation was denoted and the patient developed shoulder pain secondary to the fixed forearm malposition.

On initial presentation clinical and radiological (Figure 1) examination showed a pronounced deformity of the forearm with reduced pro- and supination of $0-20-70^{\circ}$. In comparison range of motion was $75-0-85^{\circ}$ for the right side. The wrist flexion and extension, radial and ulnar deviation, as well as elbow flexion and extension were symmetric and in the normal range of motion. Grip strength measured by Jamar was $20 \%$ less compared to the opposite side. In the clinical examination a slight laxity of the distal ulno-radial joint (DRUJ) was to the contralateral right side, with additional pronounced but painless crepitation during passive mobilization. The peripheral sensomotoric inspection was unremarkable. 


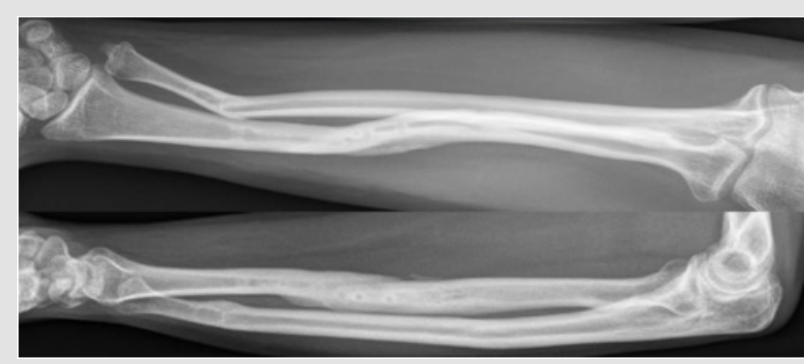

Figure 1: Anteroposterior and Lateral Pre-operative Radiographs of the Affected Forearm.

Following clinical presentation, computertomographic (CT) imaging (data $(120 \mathrm{kV}$, Philips Brilliance 40 CT, Philips Healthcare, Netherlands) of both forearms were obtained and converted to a three-dimensional (3D) triangular surface model by segmenting the bone anatomy using commercially available software (Mimics, Materialise, Belgium). Using the healthy right-side as a superimposed template (Figure 2a \& $2 b$ ) an exact quantification of the deformity could be established.

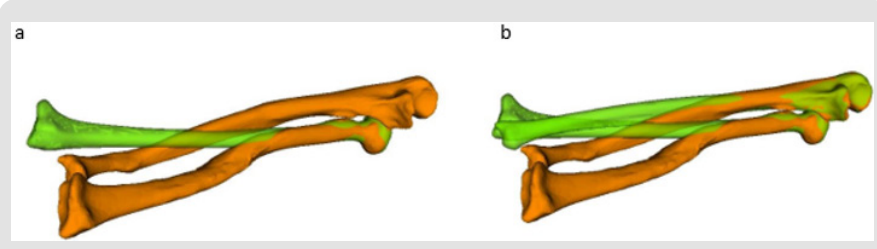

Figure 2:

a) Superimposition of the Healthy Radius (Green) Aligned with the Affected Left Radius (Orange) and

b) Aligned with Deformed Ulna and Radius.

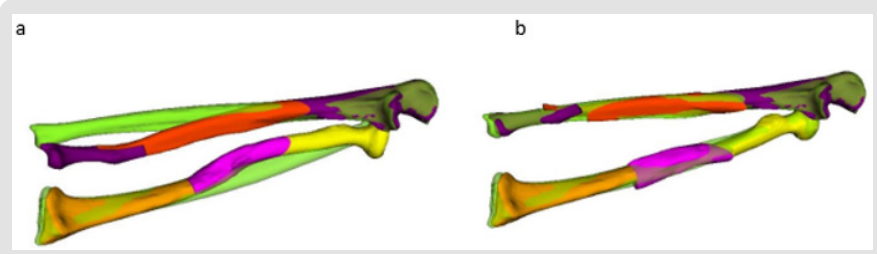

Figure 3:

a) Positioning of the Osteotomy Planes based on the Contralateral Side with

b) Simulation of Achievable Correction.

In this case the analysis revealed a pronounced malformation caused by the malunion of the fracture as well as an additional deformity due to the previous corrective osteotomy. In order to establish normal anatomic setting a four-fold corrective osteotomy of the malunion and osteotomy zone of the distal ulnar and radial shaft was needed (Figure 3a \& 3b). All four osteotomies were planned in a single plane cut manner to allow to rotate and correct the concerning fragment in the rotational axis normal to the cutting plane. This allows to fixate the fragments in plan to plane fit providing high initial stability. The final correct anatomy abolishes bony collision in pronation and thus improves range of motion. According to the anatomic restoration one large drilling and sawing guide for the radius including both osteotomies and an appropriate reduction guide was constructed whereas on the ulna each of the osteotomies had its own cutting and reposition guides (Figure 4a-4c).

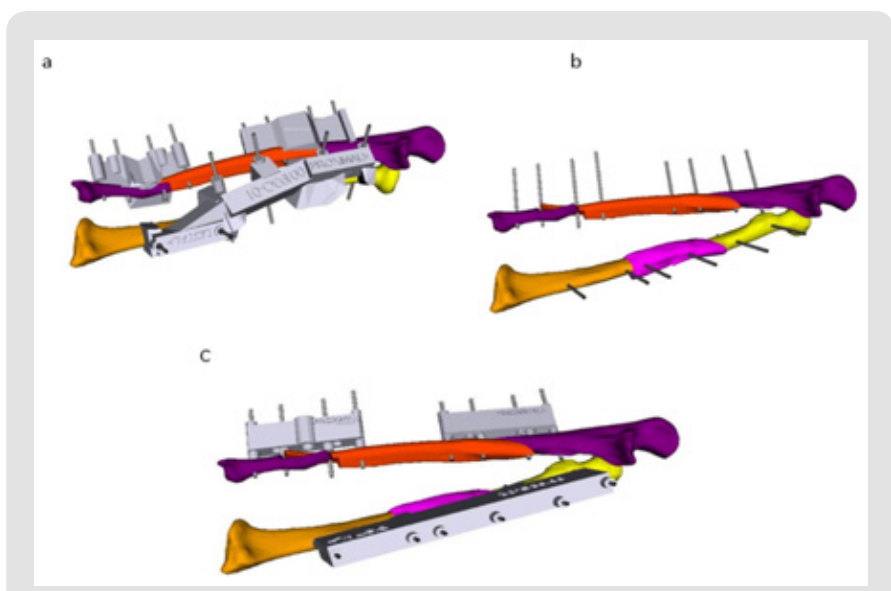

Figure 4: Guides and Simulation of Surgical Treatment.

a) Affected bones with Fitted Drilling and Cutting Guides.

b) Bone Segments in Planned Position, Following Osteotomies and Removal of Drilling and Cutting Guides.

c) Placement of Reposition Guides After Osteotomy.

\section{Surgical Procedure}

The Henry approach for the radius shaft was utilized through the interval of the brachioradial and flexor carpi radialis tendon. Primary inspection revealed the severe deformation of the radius with a ventral displacement of the supinator muscle. The resulting bumps and ridges of the radius were used for the exact fit of the guides. The supinator muscle was subsequently completely released until its insertion at the radial head; the pronator muscle could be preserved in its entirety. The large drill and saw guide, which included both osteotomies to the radius, was then placed in position. The guide was conceptualized with a gap to enable the pronator muscle to be preserved. Two K-wires of $2.0 \mathrm{~mm}$ thickness were then each placed in the middle and proximal fragment (Figure $5)$.

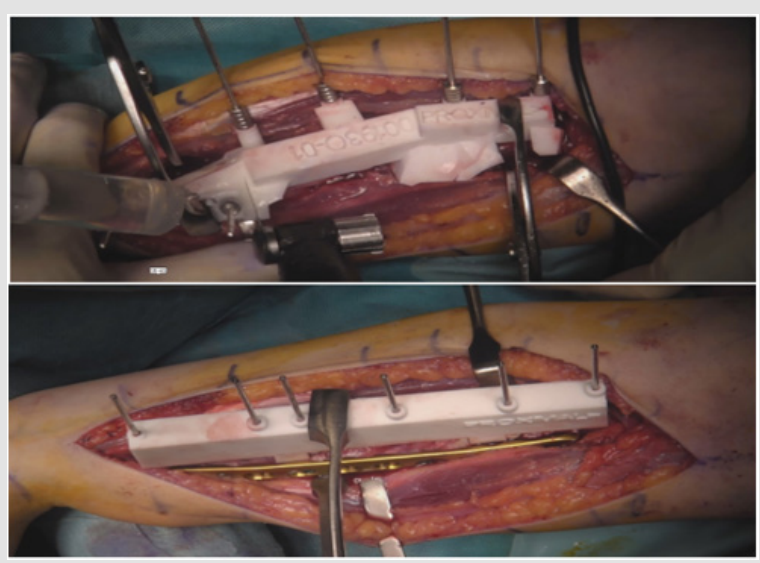

Figure 5: Intraoperative Situs of the Radius Corrective Osteotomy with Cutting and Drilling Guode Above and the Reposition Guide and Plate in Place Below. 
Subsequently the osteotomies were performed as a single-cut osteotomy. The K-wires and guide were then removed, and the $\mathrm{K}$-wires were once again inserted in the previously drilled holes. Using the wires, the middle strongly pronated fragment was supinated until all K-wires were aligned. Next, the reposition guide was placed over all wires and fragment approximation to the guide was achieved using a reposition pincer. The tentative reposition was according the pre-operative plan. Subsequently, a 10-hole $3.5 \mathrm{~mm}$ LCP Synthes plate was placed on the radial aspect of the radius. Fixation was attained with two screws in the middle and three screws in the distal fragment. An inter-fragmentary tension screw was additionally placed in the proximal osteotomy. A single unicortical screw was placed in the middle fragment. Following the removal of the guide, enough stability and increased pronation of about $40^{\circ}$ was noted.

Subsequently, the ulnar osteotomy was realized. Due to the expected better stability the proximal osteotomy was first performed. Incision was conducted dorsal of the ulnar shaft between the middle and proximal third, and the interval chosen between the extensor carpi ulnaris and flexor carpi ulnaris musculature. The drilling and sawing guide was then placed, with primary fixation with two K-wires in the distal and proximal fragment. Then, the flat oblique osteotomy was performed in a single-cut technique. The guides were then removed to allow for reposition of both fragments. The plate for ulna shortening osteotomies was placed over the four K-wires, followed by placement of the reposition guide, leading to sufficient fragment adaptation. Subsequently, angle-stable screws were inserted into the corresponding screw hole and an interfragmentary screw of $2.7 \mathrm{~mm}$ was implanted.

Now, a second incision over the previous scar was performed in order to access the distal third ulnar shaft from dorsal. Once again, the interval between the extensor carpi ulnaris and flexor carpi ulnaris musculature was used. However, due to extensive adhesion the former needed to be released. The extensively deformed ulnar shaft with an angulation of almost $40^{\circ}$ was exposed and the corresponding drill and saw guides were placed in position. The planned two K-wires were then inserted in the distal and proximal fragment. This was followed by a z-like single-cut-osteotomy, with the middle osteotomy plane corresponding to the single-cut plane. The guides were subsequently removed and the tapered ends of the osteotomy completed with the saw.

Then the derotation into anatomical position was performed and a short plate for ulna shortening osteotomy was placed over the four K-wires, followed by the reposition guide. The K-wires were then successively replaced with angle-stable screws and an additional interfragmentary screw of $2.4 \mathrm{~mm}$. The palmar protruding bone spur is flattened. Intraoperatively, an improvement in the range of motion with soft stop in both pro- and supination of around $70-0-70^{\circ}$ was denoted, whilst preserving stability of the DRUJ. Intraoperative fluoroscopy showed correct shaft positioning with an acceptable ulna-minus variance of $1.5 \mathrm{~mm}$. Subsequently, wound closure was performed, and a long wrist splint applied.

\section{Follow-up}

For eight weeks following surgery no weight bearing was permitted, but active mobilization was permitted through an occupational therapist. For adequate protection a wrist splint cast was worn during this period. Radiological examination with CT revealed correct positioning of the implant with progressive signs of consolidation in the area of the osteotomy (Figure 6a \& 6b). To further improve mobility and strength intensive occupational therapy was commenced with use of a dynamic splint. One year following four-part corrective osteotomy the patient presented with almost symmetric range of motion regarding the wrist, with a proand supination at $50-0-80^{\circ}$ (opposite side 70-0-85 ${ }^{\circ}$. Grip strength according to Jamar measured $20 \mathrm{~kg}$ (right: $22 \mathrm{~kg}$ ) and only slight discomfort could be noted during maximum forced movement. The last control at 20 months presented a pain free patient without restriction back at her profession as an occupational therapist.
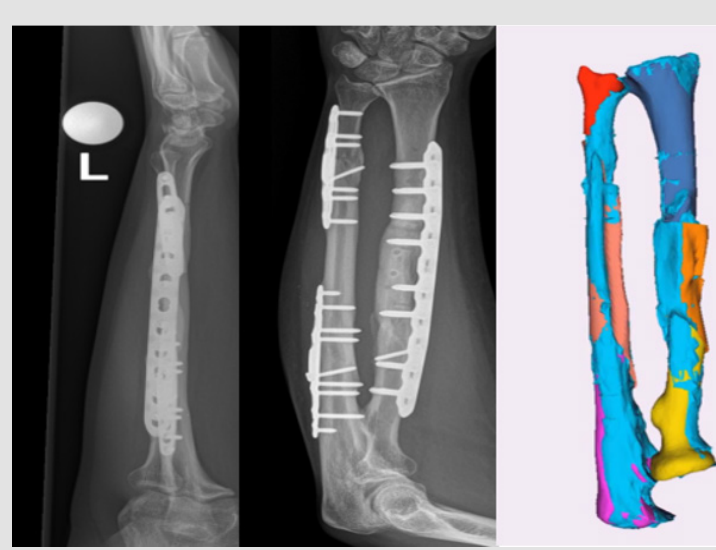

Figure 6: Anteroposterior and Lateral Radiographs (a) and (b). 3D Reconstruction (Light Blue) in Comparison with the Position of the Planning (Other Colors) at 4 Months.

\section{Discussion}

Computer-assisted 3D planning is an increasingly utilized method permitting exact quantification of the malunion and generation of patient specific guides. Whilst translation of the preoperative 3D data to correct a malunion can be challenging, comparative studies with conventional osteotomies have demonstrated favorable results regarding accuracy, surgery time and functional outcome [6,9]. The complexity of the osteotomies may vary strongly, ranging from single-cut osteotomies to multiple, composite osteotomies, in which additional rotation of fragments is used for correct anatomical positioning [10]. Particularly the preoperative estimation and intraoperative correction of a rotation has been proven more reliable compared with image intensifiercontrolled techniques. Thus, this technique particularly lends itself to the treatment of complex correction [11]. Disadvantages of this technique includes the need for specialized software, planning time, 
including guide design, and costs for the custom-made template and CT scan [9]. Nonetheless, considering the current advances in 3D printing technology patient specific custom-made guides present an effective treatment method for complex multiplanar and rotational malunions of the forearm.

\section{Conflict of Interest}

Co-founder of former CARD AG (Andreas Schweizer).

\section{Acknowledgement}

None.

\section{References}

1. Murase T, Oka K, Moritomo H, Goto A, Yoshikawa H, et al. (2008) Threedimensional corrective osteotomy of malunited fractures of the upper extremity with use of a computer simulation system. J Bone Joint Surg Am 90(11): 2375-2389.

2. Schweizer A, Philipp Fürnstahl, Matthias Harders, Gábor Székely, Ladislav Nagy (2010) Complex radius shaft malunion: osteotomy with computer-assisted planning. Hand (N Y) 5(2): 171-178.

3. McQueen M, J Caspers (1988) Colles fracture: does the anatomical result affect the final function? J Bone Joint Surg Br 70(4): 649-651.

4. Delclaux S, Trang Pham TT, Bonnevialle N, Aprédoaei C, Rongières $M$, et al. (2016) Distal radius fracture malunion: Importance of managing injuries of the distal radio-ulnar joint. Orthop Traumatol Surg Res 102(3): 327-332

ISSN: 2574-1241

DOI: 10.26717/BJSTR.2019.23.003913

Andreas Schweizer. Biomed J Sci \& Tech Res

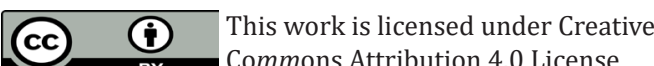

Submission Link: https://biomedres.us/submit-manuscript.php
5. Slagel BE, S Luenam, DR Pichora (2010) Management of post-traumatic malunion of fractures of the distal radius. Orthop Clin North Am 26(1): 71-84.

6. Bauer DE, Zimmermann S, Aichmair A, Hingsammer A, Schweizer A, et al. (2017) Conventional Versus Computer-Assisted Corrective Osteotomy of the Forearm: a Retrospective Analysis of 56 Consecutive Cases. J Hand Surg Am 42(6): 447-455.

7. Byrne AM, Impelmans B, Bertrand V, Van Haver A, Verstreken F (2017) Corrective Osteotomy for Malunited Diaphyseal Forearm Fractures Using Preoperative 3-Dimensional Planning and Patient-Specific Surgical Guides and Implants. J Hand Surg Am 42(10): 836e1-836e12.

8. Roner S, Vlachopoulos L, Nagy L, Schweizer A, Fürnstahl P (2017) Accuracy and Early Clinical Outcome of 3-Dimensional Planned and Guided Single-Cut Osteotomies of Malunited Forearm Bones. J Hand Surg Am 42(12): 1031e1-1031e8.

9. De Muinck Keizer RJO, Lechner KM, Mulders MAM, Schep NWL, Eygendaal D, et al. (2017) Three-dimensional virtual planning of corrective osteotomies of distal radius malunions: A systematic review and meta-analysis. Strategies Trauma Limb Reconstr 12(2): 77-89.

10. Dobbe JG, Pré KJ, Kloen P, Blankevoort L, Streekstra GJ (2011) Computerassisted and patient-specific 3-D planning and evaluation of a single-cut rotational osteotomy for complex long-bone deformities. Med Biol Eng Comput 49(12): 1363-1370.

11. Vroemen JC, Dobbe JG, Sierevelt IN, Strackee SD, Streekstra GJ (2013) Accuracy of distal radius positioning using an anatomical plate. Orthopedics 36(4): e457-62.

$\begin{array}{ll}\text { BIOMEDICAL } & \text { Assets of Publishing with us } \\ \text { RESEARCHES } & \text { - Global archiving of articles } \\ & \text { - Immediate, unrestricted online access } \\ & \text { - Rigorous Peer Review Process } \\ & \text { - Anttps://biomedres.us/ }\end{array}$

